\title{
Comunicação
}

[Communication]

\section{Perfil de ácidos graxos no leite de vacas Holandês em pastagens tropicais suplementadas com dois níveis de concentrado}

[Milk fatty acids profile of Holstein cows grazing tropical forages supplemented with two concentrate levels]

\author{
F.C.F. Lopes $^{1}$, P.A.V. Barros ${ }^{2}$, J.H. Bruschi ${ }^{3}$, P.H.F. Silva ${ }^{4}$, M.G.C.D. Peixoto ${ }^{3}$, \\ C.A.M. Gomide ${ }^{3}$, A.C.A. Duque ${ }^{2}$, M.A.S. Gama $^{3}$ \\ ${ }^{1}$ Analista, Embrapa Gado de Leite. Bolsista do CNPq. \\ ${ }^{2}$ Aluno de pós-graduação - Escola de Veterinária - UFMG - Belo Horizonte, MG \\ ${ }^{3}$ Pesquisador - Embrapa Gado de Leite \\ ${ }^{4}$ Universidade Federal de Juiz de Fora - Juiz de Fora, MG
}

\begin{abstract}
Nos últimos anos, pesquisas foram realizadas no intuito de alterar a composição da gordura do leite, tornando-a mais adequada ao consumo humano. Nesse sentido, com foco na redução do risco de doenças cardiovasculares, tem-se buscado a diminuição dos teores dos ácidos graxos saturados (AG) de cadeia média, como láurico (C12:0), mirístico (C14:0) e palmítico (C16:0), e o incremento da concentração do ácido oleico (C18:1 cis-9) no leite (Lopes et al., 2009). Devido às propriedades anticarcinogênicas, tem sido também alvo das pesquisas elevar as concentrações dos ácidos linoleicos conjugados (CLA, conjugated linoleic acid), cujo principal isômero no leite bovino é o ácido rumênico (C18:2 cis-9, trans-11), bem como de seu precursor para síntese endógena na glândula mamária, o ácido vaccênico (C18:1 trans-11) (Dewhurst et al., 2006; Lopes et al., 2009).
\end{abstract}

Em sistemas de produção de leite baseados em pastagens, é comum que a quantidade diária de concentrado seja parcelada e fornecida no cocho, depois das ordenhas. No entanto, em função da quantidade de concentrado fornecida, tal prática pode provocar alterações não desejáveis nas rotas de bio-hidrogenação ruminal dos AG da dieta e na composição e perfil de AG do leite (Dewhurst et al., 2006).

Recebido em 28 de setembro de 2010

Aceito em 1 de março de 2011

E-mail: fernando@cnpgl.embrapa.br

Trabalho financiado pela FAPEMIG
Na literatura consultada, não foram encontrados trabalhos de avaliação do efeito da suplementação concentrada sobre o perfil de AG no leite de vacas em pastagens tropicais, mas o foram em pastejo com forrageiras de clima temperado (Bargo et al., 2006). Os poucos resultados disponíveis em pastagens tropicais foram obtidos a partir da utilização de concentrados ricos em AG (Medeiros et al., 2010), o que impede a avaliação do potencial per se da forrageira tropical na obtenção de leite com perfil de AG mais adequado ao consumo humano e, por conseguinte, valorização da qualidade nutricional diferenciada do leite produzido em sistemas de produção baseados em pastagens tropicais.

O experimento foi realizado em dezembro de 2008 na Embrapa Gado de Leite (Coronel Pacheco, MG), com o objetivo de avaliar o perfil de AG no leite de vacas da raça Holandês mantidas em pastagens formadas com as gramíneas tropicais Brachiaria brizantha cV. xaraés e Panicum maximum cv. tanzânia, suplementadas com 3 ou $6 \quad \mathrm{~kg} / \mathrm{dia}$ de concentrado.

Foram utilizadas 12 vacas, com média de produção de $15 \mathrm{~kg}$ de leite/dia (4,0\% de gordura; $3,4 \%$ de proteína; $4,0 \%$ de lactose e $12,8 \%$ de extrato seco total), em delineamento de blocos ao 
acaso, em esquema fatorial $2 \times 2$, com dois níveis de suplementação concentrada (3 ou 6 $\mathrm{kg} / \mathrm{vaca} / \mathrm{dia}$ ) e duas espécies de gramíneas, tanzânia e xaraés - que apresentaram a seguinte composição química média (\% da MS), respectivamente: 10,0 e 9,6\% de proteína bruta (PB); 69,4 e 71,1\% de fibra em detergente neutro (FDN); 1,4 e 1,7\% de extrato etéreo (EE); a digestibilidade in vitro da MS (DIVMS) foi de 46,7 e 50,6\%, respectivamente.

As pastagens foram manejadas em sistema de lotação intermitente, com 30 dias de intervalo de desfolha e três de ocupação dos piquetes. O concentrado (87,7\% de MS; 85,2\% de DIVMS; $23,9 \%$ de $\mathrm{PB}$ e 1,9 \% de $E E)$ não possuía nenhum ingrediente rico em $\mathrm{AG}$, tendo sido formulado com $60 \%$ de fubá de milho, $35 \%$ de farelo de soja, $2 \%$ de mistura mineral-vitamínica, $1 \%$ de calcáreo calcítico, $1 \%$ de ureia, e 1\% de bicarbonato de potássio, sendo fornecido às vacas em cocho individual, parcelado em duas vezes, após as ordenhas (seis e 14 h).

Durante seis dias consecutivos, foram coletadas amostras de leite, em frascos sem conservante, que foram armazenadas a $-10^{\circ} \mathrm{C}$, visando à determinação do perfil de AG por cromatografia em fase gasosa, utilizando-se coluna capilar de sílica fundida (CPSil-88, 100m x 0,25mm x $0,2 \mu \mathrm{m})$ e detector de ionização de chama, conforme procedimentos descritos por CruzHernandez et al. (2007).

Os efeitos dos fatores forragem, suplementação concentrada e interação entre eles foram analisados pelo procedimento MIXED do SAS (2002). Quando a interação foi significativa $(\mathrm{P}<0,05)$, as variáveis de perfil de AG do leite foram analisadas por contrastes ortogonais pelo procedimento GLM do SAS (2002).

A interação de fatores forrageira e nível de suplementação concentrada foi significativa $(\mathrm{P}<0,05)$ apenas para os AG C6:0, C10:0, C12:0, C13:0 e C18:1 cis-9. O efeito de forragem foi significativo $(\mathrm{P}<0,05)$ para os teores dos AG C18:1 trans-9, C18:1 trans-10 e $\alpha$-linolênico (C18:3 cis-9, cis-12, cis-15). Não foram encontrados, na literatura consultada, trabalhos com perfil comparativo de AG em gramíneas tropicais, mas, de modo geral, os principais AG presentes são palmítico, linoleico e $\alpha$-linolênico, havendo, no entanto, importantes diferenças, quanto à concentração deles, entre espécies forrageiras (Comunicação pessoal: Antoniassi, R.,10/06/10, Embrapa Agroindústria de Alimentos, Rio de Janeiro, RJ). Assim, eventuais diferenças no teor do AG $\alpha$-linolênico poderiam justificar o efeito observado de forrageira $(\mathrm{P}<0,05)$ sobre a concentração deste AG no leite das vacas em pastagens de xaraés e tanzânia $(0,35$ e $0,46 g / 100 g$ de AG totais, respectivamente). $\quad \mathrm{Na}$ suplementação concentrada, houve efeito $(\mathrm{P}<0,05)$ apenas para o AG $\alpha$-linolênico (Tab. 1), cujas concentrações foram maiores no leite das vacas que consumiram menor quantidade de concentrado $(0,45$ e $0,36 \mathrm{~g} / 100 \mathrm{~g}$ de AG totais, respectivamente, para 3 e $6 \mathrm{~kg} / \mathrm{vaca} / \mathrm{dia})$. É possível que, na suplementação mais alta (6kg/vaca/dia), tenha havido maior taxa de substituição do consumo de pasto, levando ao menor consumo de AG $\alpha$-linolênico e, por conseguinte, maior concentração deste AG no leite das vacas que receberam $3 \mathrm{~kg} / \mathrm{dia}$ de concentrado, conforme sugeriram Bargo et al. (2006). Entretanto, ressalta-se que não houve efeito $(\mathrm{P}>0,05)$ sobre o teor de ácido vaccênico no leite (Tab. 1), o qual é intermediário comum dos processos de bio-hidrogenação tanto do AG $\alpha$-linolênico como do linoleico (Collomb et al., 2006).

As concentrações médias dos AG rumênico e vaccênico foram, respectivamente, de 1,01 e $1,79 \mathrm{~g} / 100 \mathrm{~g}$ de $\mathrm{AG}$ totais $(\mathrm{P}>0,05 ;$ Tab. 1$)$. Dewhurst et al. (2006) relataram que as concentrações de CLA no leite de vacas que receberam forrageiras de clima temperado, fornecidas frescas ou conservadas, variaram, respectivamente, de 0,72 a 2,64 e de 0,27 a 0,68g/100g de AG totais. Elgersma et al. (2006) observaram que a concentração de CLA e ácido vaccênico no leite de vacas em pastagens de clima temperado variaram de 0,11 a 2,43 e de 0,66 a 4,62g/100g de AG, respectivamente. Os valores obtidos para estes AG estão dentro das amplitudes de variação relatadas por esses autores, para forrageiras de clima temperado fornecidas frescas, e demonstram o potencial das forrageiras tropicais na produção de leite com níveis superiores de CLA, pois, no presente trabalho, o concentrado oferecido não continha ingredientes ricos em AG $\alpha$-linolênico e linoleico, precursores para bio-hidrogenação ruminal. 
Lopes et al.

Tabela 1. Perfil de ácidos graxos (g/100g de ácidos graxos totais) no leite de vacas da raça Holandês mantidas em dois tipos de pastagem (For: tanzânia ou xaraés) e suplementadas com dois níveis de concentrado (Conc: 3 ou 6 kg/vaca/dia)

\begin{tabular}{|c|c|c|c|c|c|c|c|c|}
\hline \multirow{3}{*}{ Ácido graxo } & \multicolumn{4}{|c|}{ Tratamento } & \multirow{3}{*}{$\begin{array}{l}\text { CV } \\
(\%)\end{array}$} & \multicolumn{3}{|c|}{ Efeito } \\
\hline & \multicolumn{2}{|c|}{ Tanzânia } & \multicolumn{2}{|c|}{ Xaraés } & & For & Conc & For*Conc \\
\hline & 3kg/dia & $6 \mathrm{~kg} / \mathrm{dia}$ & 3kg/dia & $6 \mathrm{~kg} / \mathrm{dia}$ & & \multicolumn{3}{|c|}{ Valor de $\mathrm{P}$} \\
\hline $\bar{C} 4: 0$ & 3,02 & 2,97 & 2,88 & 3,02 & 10,2 & $\mathrm{~ns}^{\mathrm{c}}$ & ns & ns \\
\hline C6:0 & 2,01 & 1,75 & 1,81 & 2,00 & 7,1 & ns & ns & 0,03 \\
\hline C8:0 & 1,17 & 0,92 & 1,01 & 0,68 & 29,3 & ns & ns & ns \\
\hline C10:0 & 2,46 & 1,77 & 2,16 & 2,48 & 10,8 & ns & ns & 0,01 \\
\hline C11:0 & 0,39 & 0,33 & 0,34 & 0,42 & 16,6 & ns & ns & ns \\
\hline C12:0 & 2,97 & 2,17 & 2,57 & 2,92 & 12,2 & ns & ns & 0,02 \\
\hline C13:0 & 0,10 & 0,07 & 0,09 & 0,16 & 28,6 & ns & ns & 0,02 \\
\hline C14:0 & 10,7 & 8,88 & 9,75 & 9,74 & 7,2 & ns & ns & ns \\
\hline C14:1 cis-9 & 1,20 & 1,14 & 1,27 & 1,22 & 18,9 & ns & ns & ns \\
\hline C15:0 & 1,18 & 1,10 & 1,03 & 1,12 & 10,6 & ns & ns & ns \\
\hline C16:0 & 28,1 & 26,7 & 26,6 & 28,9 & 7,4 & ns & ns & ns \\
\hline C16:1 cis-9 & 1,57 & 1,57 & 1,78 & 1,42 & 24,4 & ns & ns & ns \\
\hline C17:0 & 0,52 & 0,39 & 0,54 & 0,42 & 39,2 & ns & ns & ns \\
\hline C18:0 & 10,5 & 11,6 & 12,0 & 11,4 & 13,9 & ns & ns & ns \\
\hline C18:1 trans- 6 a trans-8 & 0,15 & 0,18 & 0,19 & 0,49 & 70,7 & ns & ns & ns \\
\hline C18:1 trans-9 & 0,26 & 0,28 & 0,30 & 0,38 & 13,0 & 0,03 & 0,07 & ns \\
\hline C18:1 trans -10 & 0,21 & 0,25 & 0,34 & 0,36 & 20,2 & 0,01 & ns & ns \\
\hline C18:1 trans-11 & 1,64 & 1,66 & 1,90 & 2,04 & 17,7 & ns & ns & ns \\
\hline C18:1 trans-12 & 0,21 & 0,19 & 0,19 & 0,17 & 17,8 & ns & ns & ns \\
\hline C18:1 trans-13 e trans-14 & 0,58 & 0,50 & 0,63 & 0,72 & 24,7 & ns & ns & ns \\
\hline C18:1 cis-9 & 20,9 & 25,5 & 22,4 & 20,8 & 9,1 & ns & ns & 0,05 \\
\hline C18:2 cis-9, cis-12 & 1,07 & 1,35 & 1,22 & 1,16 & 14,2 & ns & ns & ns \\
\hline C18:3 cis-9, cis-12, cis-15 & 0,49 & 0,42 & 0,40 & 0,30 & 8,4 & 0,001 & 0,002 & ns \\
\hline CLA cis-9, trans-11 & 0,91 & 1,10 & 1,01 & 1,04 & 21,4 & ns & ns & ns \\
\hline CLA trans-9, cis-11 & 0,04 & 0,05 & 0,03 & 0,02 & 50,2 & ns & 0,08 & ns \\
\hline C20:0 & 0,11 & 0,13 & 0,13 & 0,08 & 51,0 & ns & ns & ns \\
\hline
\end{tabular}

$\overline{\mathrm{CV}}=$ coeficiente de variação; For: efeito forrageira; Conc: efeito concentrado; ns= não significativo $(\mathrm{P}>0,05)$.

Trabalhando com a gramínea tropical capimelefante (Pennisetum purpureum) fornecida picada no cocho na forma de dieta completa, misturada ao concentrado, Lopes et al. (2009) relataram concentrações dos AG oleico, vaccênico e rumênico, respectivamente, de 20,4; 2,00 e 1,28g/100g de AG totais no leite de vacas Holandês x Zebu, valores próximos aos da Tab. 1. A soma das concentrações dos AG C12:0, C14:0 e C16:0, que têm sido associados à incidência de doenças coronárias, apresentada por esses autores, foi de 44,8g/100g de AG totais, maior que a do presente estudo (39,8g/100g de AG totais), demonstrando que o leite produzido sob condição de pastejo em gramíneas tropicais apresentou perfil de AG menos aterogênico e colesterolêmico.
Os resultados obtidos ilustram o potencial de gramíneas tropicais manejadas sob pastejo na produção de leite com perfil de AG reconhecidamente mais saudável em termos da nutrição e saúde humana. Tal informação é de extrema importância para a indústria de laticínios do ponto de vista tecnológico, e na prospecção e fomento de nichos de mercados interno ou externo para comercialização, bem como para definição de critérios e eventual implementação de políticas de bonificação de pagamento ao empreendedor rural que produza leite com tais características nutracêuticas.

Palavras-chave: ácido linoleico conjugado, Brachiaria brizantha, Panicum maximum, vaca leiteira 


\begin{abstract}
Milk fatty acids profile was evaluated using 12 Holstein cows producing $15 \mathrm{~kg} /$ day of milk with $4.0 \%$ of fat. The animals were assigned to four treatments in a $2 \times 2$ factorial arrangement (two tropical grasses Brachiaria brizantha $c v$. Xaraés and Panicum maximum cv. Tanzania, supplemented with two concentrate levels -3 and $6 \mathrm{~kg} / \mathrm{cow} /$ day) in a completely randomized block design. There was no effect of forage and concentrate level $(P>0.05)$ on concentrations of rumenic, vaccenic, (C18: 1 trans-11) and oleic acids in milk fat (mean values of 1.01, 1.79, and $22.55 \mathrm{~g} / 100 \mathrm{~g}$ of total fatty acids, respectively). The concentrations of lauric (C12:0), myristic (C14:0), and palmitic (C16:0) acids were also unaffected by treatments (mean values of 2.63, 9.77, and $27.44 \mathrm{~g} / 100 \mathrm{~g}$ of total fatty acids, respectively). Overall, the results illustrate the potential of tropical grasses in producing milk containing a more desirable fatty acids profile in terms of human health.
\end{abstract}

Keywords: dairy cow, conjugated linoleic acid, Brachiaria brizantha, Panicum maximum

\section{REFERÊNCIAS BIBLIOGRÁFICAS}

BARGO, F.; DELAHOY, J.E.; SCHROEDER, G.F. et al. Milk fatty composition of dairy cows grazing at pasture allowances and supplement with different levels ans sources of concentrate. Anim. Feed Sci. Technol., v.125, p.17-31, 2006.

COLLOMB, M.; SCHMID, A.; SIEBER, R. et al. Conjugated linoleic acids in milk fat: variation and physiological effects. Int. Dairy J., v.16, p.1347-1361, 2006.

CRUZ-HERNANDEZ, J.K.G.; KRAMER, J.J.; KENNELLY, D.R. et al. Evaluating the conjugated linoleic acid and trans18:1 isomers in milk fat of dairy cows fed increasing amounts of sunflower oil and a constant level of fish oil. $J$. Dairy Sci., v.90, p.3786-3801, 2007.

DEWHURST, R.J.; SHINGFIELD, K.J.; LEE, M.R.F. et al. Increasing the concentrations of beneficial polyunsaturated fatty acids in milk produced by dairy cows in high-forage systems. Anim. Feed Sci. Technol., v.131, p.168-206, 2006.
ELGERSMA, A.; TAMMINGA, S.; ELLEN, G. Modifying milk composition through forage. Anim. Feed Sci. Technol., v.131, p.207-225, 2006.

LOPES, F.C.F.; RIBEIRO, C.G.S.; RIBEIRO, M.T. et al. Milk fatty acid profile from dairy cows fed increasing levels of soybean oil in diets based on tropical forage. In: INTERNATIONAL SYMPOSIUM ON RUMINANT PHYSIOLOGY, 11., 2009, Clermont-Ferrand. Proceedings... Clermont-Ferrand: INRA, 2009.

MEDEIROS, S.R.; OLIVEIRA, D.E.; AROEIRA, L.J.M. et al. Effects of dietary supplementation of rumen-protected conjugated linoleic acid to grazing cows in early lactation, $J$. Dairy Sci., v.93, p.1126-1137, 2010. 